\title{
Recurrent Lentigo Maligna Melanoma
}

National Cancer Institute

\section{Source}

National Cancer Institute. Recurrent Lentigo Maligna Melanoma. NCI Thesaurus. Code C8785.

The reemergence of lentigo maligna after a period of remission 